\title{
New traveling wave solutions of MHD micropolar fluid in porous medium
}

\author{
Muhammad Jamil ${ }^{*}$ and Arsalan Ahmed
}

*Correspondence:
jqrza@neduet.edu.pk;
jqrza26@yahoo.com
Department of Mathematics, NED
University of Engineering \&
Technology, Karachi 75270, Pakistan

Technology, Karachi 75270, Pakistan

\begin{abstract}
This paper aims to determine the exact solutions of non-Newtonian fluid namely micropolar fluid with MHD in a porous medium by traveling wave method. The governing equations of incompressible micropolar fluid with MHD in a porous medium are non-linear PDEs reduced to ODEs through wave parameter $\xi=m x+n y+U t$. The set of new exact solutions are determined for five different cases. In special cases, the solution for micropolar fluid with and without MHD and porous effects can also be obtained from general solutions. Furthermore, these solution reduces to a Newtonian solution if we put vortex viscosity $\kappa \rightarrow 0$. Finally, the influence of the material and other parameters of interest on the fluid motion, as well as a comparison among micropolar and Newtonian fluids is also analyzed by 2D and 3D graphical illustrations.
\end{abstract}

Keywords: Traveling wave, MHD, Micropolar fluid, Porous medium, Exact solutions, 2D \& 3D graphs

Mathematics Subject Classification (2010): 76A05, 76A10

\section{Introduction}

In the present, many researchers are working on the non-Newtonian fluid from both essential and sensible point of view [1]. These fluids have immediate effects on the processing of polymer, animal blood, liquid crystal, and geological flows in the earth mantle. The general conditions of non-Newtonian fluid are exceptionally non-linear and higherarrangement than Navier-Stokes equations. Therefore, many analytical and numerical solutions are accessible to non-Newtonian fluid on the topic.

The electrically conducting fluid and magnetic properties are sufficiently studied in magnetohydrodynamics (MHD). MHD has specific applications like engineering science, metallurgical industry, electromagnetic pump, power generation, and flow meter. Hydromagnetic movements have a major part of study in the fields of the aerospace, astronomical, and planetary magnetosphere. The cleansing of liquid metals from non-metallic presence through the use of the attractive field is another basic component of MHD [2, 3]. Khalid et al. [4] have evaluated the exact solution of wall couple stress in MHD by Laplace transform and convolution. Fatunmbi et al. [5] have numerically studied the MHD stagnation point flow of a micropolar fluid by applying RK (Runge-Kutta) method. Hammouch [6] investigated the numerous solution of steady magnetohydrodynamics flow of dilatant

(C) The Author(s). 2020 Open Access This article is licensed under a Creative Commons Attribution 4.0 International License which permits use, sharing, adaptation, distribution and reproduction in any medium or format, as long as you give appropriate credit to the original author(s) and the source, provide a link to the Creative Commons licence, and indicate if changes were made. The images or other third party material in this article are included in the article's Creative Commons licence, unless indicated otherwise in a credit line to the material. If material is not included in the article's Creative Commons licence and your intended use is not permitted by statutory regulation or exceeds the permitted use, you will need to obtain permission directly from the copyright holder. To view a copy of this licence, visit http://creativecommons.org/licenses/by/4.0/. 
fluids by various method techniques. Interesting recent studies on non-newtonian fluids with magnetohydrodynamics have been given by different researchers [7-11].

Micropolar fluid theory was proposed by Eriggen [12]. Mekheimer et al. [13] showed that the micropolar fluid model is a kind of non-Newtonian fluid which depends upon a microstructure and belongs to non-symmetrical stress tensor. Physically, micropolar fluid may be rigid particles, at random oriented (spherical) elements suspended in a viscous medium where the change of fluid elements is disregarded. Micropolar fluid can perform a better model for animal blood. Raza et al. [14] studied the different branches of the solution of micropolar fluid in a channel with permeable walls. Hussanan et al. [15] examined the heat and mass transfer in a micropolar fluid. Sheikholeslami et al. [16] evaluated the micropolar fluid flow in a channel by homotopy perturbation method (HPM). Lukaszewicz [17] explored the nature of the micropolar fluid. Ravi and Prasad [18] studied the relations of pulsatile and peristaltic stream of couple stress fluid through a porous medium in an elastic route.

There are many methods for solving non-linear partial differential equations such as bilinear transformation $[19,20]$, homotopy perturbation method [21, 22], $\left(G^{\prime} \backslash G\right)$ expansion method [23], Exp-function [24, 25], modified simple equation (MSE) method [26], and fingero-imbibition method [27]. The traveling wave method is useable for solving non-linear partial differential equations because it provides a simple exact solution of non-linear partial differential equations. Due to the many importance of this method in theory of non-linear partial differential equations, this method is applicable in the field of fluid mechanics, chemical kinematics, electromagnetic, and nonlinear mechanics. Some important work-related traveling wave solutions recently appeared in [28-34] and references presented there.

In the present communication, the traveling wave solution, and transformation method are used to find the exact solutions of unsteady incompressible two-dimensional laminar flow of MHD micropolar fluid in a porous medium. The mathematical model that considered here are carefully entrenched and judge with many general cases. It is noticed that the present model is capable of covering such benchmark cases and can give new information on the parameter determination of the dynamical system which governed micropolar fluid equations. To the best of our knowledge, only one study, which deals with the flows of unsteady incompressible two-dimensional laminar flow of micropolar fluid via traveling wave transformation appeared in [28]. Therefore, this communication aims to extend the results of [28] for unsteady two-dimensional incompressible laminar flow of MHD micropolar fluid in a porous medium. The family of new exact solutions is found for five distinct cases. Particularly, the solutions for micropolar fluid with and without MHD and porous effects can also be established from present general solutions. The solutions for MHD Newtonian fluid in the porous medium may for all cases easily be determined if we substitute vortex viscosity $\kappa \rightarrow 0$ in respective equations. Finally, the impact of parameters defines micropolar fluid and other parameters are analyzed through 2D and 3D graphical illustrations and differences among micropolar and Newtonian fluids are also discussed. For the present paper, we adopted the method [28-33] in the following pattern. In the "Basic governing equations" section, we provide the basic governing equations and the theoretical development of the traveling wave method. In the "Traveling wave solutions" section, we present the solution of our governing equations by the traveling wave methods along with five different cases for MHD micropolar fluid in a porous medium. 
Some initial and boundary conditions are discussed in the "Making initial and boundary conditions" section. In the "Numerical results and discussions" section, we discuss the numerical results with the help of graphical illustrations.

\section{Basic governing equations}

The equation of continuity and the conservation equations of linear momentum and angular momentum for an incompressible unsteady micropolar fluid, in the presence of magnetohydrodynamic through a porous medium, by neglecting the body force and body couple are as follows:

$$
\begin{aligned}
\nabla \cdot \vec{V} & =0, \\
\rho \frac{D \vec{V}}{D t} & =-\nabla p+(\mu+\kappa) \nabla^{2} \vec{V}+\kappa \nabla \times \vec{\Omega}+\vec{J} \times \vec{B}-\left(\frac{v}{k^{*}}\right) \vec{V}, \\
\rho j \frac{D \vec{\Omega}}{D t} & =(\alpha+\beta+\gamma) \nabla(\nabla \cdot \vec{\Omega})-\gamma \nabla \times(\nabla \times \vec{\Omega})+\kappa \nabla \times \vec{V}-2 \kappa \vec{\Omega} .
\end{aligned}
$$

In the above equations, $\vec{V}$ is a velocity; $\vec{\Omega}$ is a microrotation; $p$ be the pressure; $\rho$ is the fluid density; $j$ be the gyration parameters of the fluid; $\mu$ and $\kappa$ are the dynamic and vortex viscosities; $\alpha, \beta$, and $\gamma$ are the respective coefficients of coupled viscosities [29]; $\vec{B}$ is the total magnetic force vector; $\vec{J}$ is the current density; and $\kappa^{*}$ is the porosity parameter.

The velocity, microrotation, and magnetic component porous plate are $(u, v, 0),(0,0, N)$, and $\left(0,0, B_{o}\right)$, respectively, where $u$ and $v$ are the second and third components of velocity, respectively, and $N$ is the second component of microrotation while $\vec{B}$ is total magnetic filed, so that $\vec{B}=\overrightarrow{B_{0}}+\vec{b}, \vec{b}$ is the induced magnetic filed and using $\vec{J} \times \vec{B}=-\sigma B_{o} \vec{V}$, where $\sigma$ is the electrically conductively of fluid and then Eqs. (1-3) provide the following governing equations (see Fig. 1):

$$
\begin{aligned}
\frac{\partial u}{\partial x}+\frac{\partial v}{\partial y} & =0 \\
\frac{\partial u}{\partial t}+u \frac{\partial u}{\partial x}+v \frac{\partial u}{\partial y} & =-\frac{\partial p}{\partial x}+\left(v+\frac{\kappa}{\rho}\right)\left(\frac{\partial^{2} u}{\partial x^{2}}+\frac{\partial^{2} u}{\partial y^{2}}\right)+\frac{\kappa}{\rho} \frac{\partial N}{\partial y}-\left(\frac{\sigma B_{o}^{2}}{\rho}+\frac{v}{k^{*}}\right) u,
\end{aligned}
$$

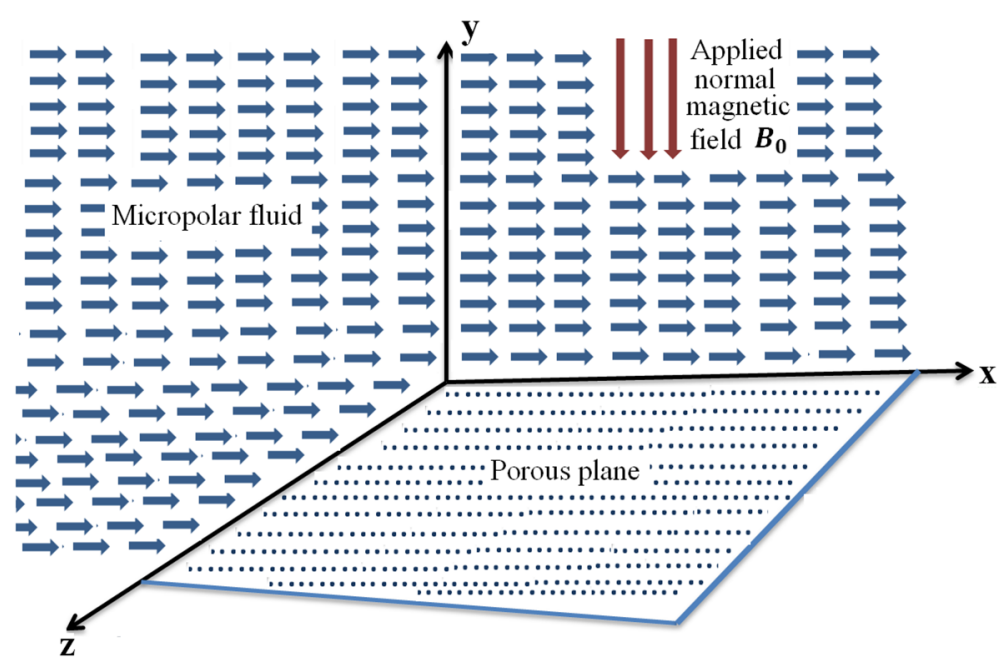

Fig. 1 Two dimensional flow of micropolar fluid in the presence of normal magnetic field on porous plane 


$$
\begin{gathered}
\frac{\partial v}{\partial t}+u \frac{\partial v}{\partial x}+v \frac{\partial v}{\partial y}=-\frac{\partial p}{\partial y}+\left(v+\frac{\kappa}{\rho}\right)\left(\frac{\partial^{2} v}{\partial x^{2}}+\frac{\partial^{2} v}{\partial y^{2}}\right)-\frac{\kappa}{\rho} \frac{\partial N}{\partial x}-\left(\frac{\sigma B_{o}^{2}}{\rho}+\frac{v}{k^{*}}\right) v \\
\frac{\partial N}{\partial t}+u \frac{\partial N}{\partial x}+v \frac{\partial N}{\partial y}=\frac{\gamma}{\rho j}\left(\frac{\partial^{2} N}{\partial x^{2}}+\frac{\partial^{2} N}{\partial y^{2}}\right)-\frac{\kappa}{\rho j}\left(2 N-\frac{\partial v}{\partial x}+\frac{\partial u}{\partial y}\right) .
\end{gathered}
$$

\section{Procedure of traveling wave method}

Suppose the non-linear partial differential equations include four dependent variables $u, v, N$, and $p$ three variables $t, x$, and $y$.

$$
\begin{array}{r}
M_{1}\left(u_{x}, v_{y}\right)=0, \\
M_{2}\left(u, v, u_{t}, u_{x}, u_{y}, u_{x x}, u_{y y}, N_{y}, p_{x}\right)=0, \\
M_{3}\left(u, v, v_{t}, v_{x}, v_{y}, v_{x x}, v_{y y}, N_{x}, p_{y}\right)=0, \\
M_{4}\left(N_{t}, N_{x}, N_{y}, N_{x x}, N_{y y}, N, u_{y}, u_{x}\right)=0 .
\end{array}
$$

Here, $M_{i}$ is a polynomial function of $u, v, N$, and $p$ which contain the non-linear terms and higher order of derivative; where $i=1,2,3,4$., we display traveling wave solution as follows:

$$
u(x, y, t)=u(\xi), v(x, y, t)=v(\xi), N(x, y, t)=N(\xi), p(x, y, t)=p(\xi),
$$

where $\xi=m x+n y+U t$. The system Eqs. (8-11) can be converted into the system of ordinary differential equations.

$$
\begin{aligned}
M_{1}\left(u^{\prime}, v^{\prime}\right) & =0, \\
M_{2}\left(u, v, u^{\prime}, u^{\prime \prime}, N^{\prime}, p^{\prime}\right) & =0, \\
M_{3}\left(u, v, v^{\prime}, v^{\prime \prime}, N^{\prime}, p^{\prime}\right) & =0, \\
M_{4}\left(N, N^{\prime}, N^{\prime \prime}, u^{\prime}, v^{\prime}\right) & =0 .
\end{aligned}
$$

This system of ordinary differential equations are may or may not be solvable. In our case, these system are solvable.

\section{Traveling wave solutions}

In this section, we will determine the traveling wave solutions of governing Eqs. (4-7). The traveling wave parameter type can be taken, and then, the solution has the following representation.

$$
u=u(\xi), v=v(\xi), N=N(\xi), p=p(\xi) \text { and } \xi=m x+n y+U t .
$$

Here, $U$ is the constant phase velocity and $m$ and $n$ are constants. On using the traveling wave parameter $\xi$ into Eqs. (4-7), we get representation of the form

$$
\begin{aligned}
m u^{\prime}+n v^{\prime} & =0 \\
(U+m u+n v) u^{\prime} & =-m p^{\prime}+\left(v+\frac{\kappa}{\rho}\right)\left(m^{2}+n^{2}\right) u^{\prime \prime}+\frac{n \kappa}{\rho} N^{\prime}-\left(\frac{\sigma B_{o}^{2}}{\rho}+\frac{v}{k^{*}}\right) u,
\end{aligned}
$$




$$
\begin{gathered}
(U+m u+n v) v^{\prime}=-n p^{\prime}+\left(v+\frac{\kappa}{\rho}\right)\left(m^{2}+n^{2}\right) v^{\prime \prime}-\frac{m \kappa}{\rho} N^{\prime}-\left(\frac{\sigma B_{o}^{2}}{\rho}+\frac{v}{k^{*}}\right) v, \\
(U+m u+n v) N^{\prime}=\frac{\gamma}{\rho j}\left(m^{2}+n^{2}\right) N^{\prime \prime}-\frac{\kappa}{\rho j}\left(2 N-m v^{\prime}+n u^{\prime}\right),
\end{gathered}
$$

where the prime denotes the differentiation with respect to $\xi$. Integration to Eq. (17), w.r.t $\xi$ on both side yields

$$
m u+n v=c_{0}
$$

where $c_{0}$ is an arbitrary constant. Using Eq. (21) in Eqs. (18)-(20) reduced to

$$
\begin{gathered}
\left(U+c_{0}\right) u^{\prime}=-m p^{\prime}+\left(v+\frac{\kappa}{\rho}\right)\left(m^{2}+n^{2}\right) u^{\prime \prime}+\frac{n \kappa}{\rho} N^{\prime}-\left(\frac{\sigma B_{o}^{2}}{\rho}+\frac{v}{k^{*}}\right) u \\
\left(U+c_{0}\right) v^{\prime}=-n p^{\prime}+\left(v+\frac{\kappa}{\rho}\right)\left(m^{2}+n^{2}\right) v^{\prime \prime}-\frac{m \kappa}{\rho} N^{\prime}-\left(\frac{\sigma B_{o}^{2}}{\rho}+\frac{v}{k^{*}}\right) v \\
\left(U+c_{0}\right) N^{\prime}=\frac{\gamma}{\rho j}\left(m^{2}+n^{2}\right) N^{\prime \prime}-\frac{\kappa}{\rho j}\left(2 N-m v^{\prime}+n u^{\prime}\right) .
\end{gathered}
$$

To find $u, v, N$, and $p$ from the above three equations, the following five cases will be discussed.

\section{Case-I: $U+c_{0} \neq 0$}

Eliminating $p$ from Eqs. (22) and (23), we get

$$
\begin{aligned}
\left(U+c_{0}\right)\left(n u^{\prime}-m v^{\prime}\right)= & \left(m^{2}+n^{2}\right)\left(v+\frac{\kappa}{\rho}\right)\left(n u^{\prime \prime}-m v^{\prime \prime}\right)+\frac{\kappa}{\rho}\left(m^{2}+n^{2}\right) N^{\prime} \\
& -\left(\frac{\sigma B_{o}^{2}}{\rho}+\frac{v}{k^{*}}\right)(n u-m v) .
\end{aligned}
$$

Again, using Eq. (21) and converting $v$ into $u$ in Eq. (25), we arrive

$$
\begin{gathered}
N^{\prime}=\frac{\rho}{\kappa}\left[\frac{1}{n}\left(U+c_{o}\right) u^{\prime}-\frac{1}{n}\left(m^{2}+n^{2}\right)\left(v+\frac{\kappa}{\rho}\right) u^{\prime \prime}+\frac{1}{n}\left(\frac{\sigma B_{o}^{2}}{\rho}+\frac{v}{k^{*}}\right) u\right. \\
\left.-\frac{m c_{o}}{n\left(m^{2}+n^{2}\right)}\left(\frac{\sigma B_{o}^{2}}{\rho}+\frac{v}{k^{*}}\right)\right] .
\end{gathered}
$$

Eliminating $N$ from Eqs. (24) and (26), we get

$$
a_{1} u^{i \mathrm{v}}-a_{2} u^{\prime \prime \prime}+a_{3} u^{\prime \prime}+a_{4} u^{\prime}+a_{5} u=a_{6},
$$

where

$$
\begin{aligned}
& a_{1}=\frac{\gamma}{n j \kappa}\left(m^{2}+n^{2}\right)^{2}\left(v+\frac{\kappa}{\rho}\right), \\
& a_{2}=\left[\frac{\rho}{n \kappa}\left(U+c_{0}\right)\left(m^{2}+n^{2}\right)\left(v+\frac{\kappa}{\rho}\right)+\frac{\gamma}{n j \kappa}\left(m^{2}+n^{2}\right)\left(U+c_{o}\right)\right], \\
& a_{3}=\left[\frac{\rho}{n \kappa}\left(U+c_{0}\right)^{2}+\frac{\kappa}{n j \rho}\left(m^{2}+n^{2}\right)-\frac{\gamma}{n j \kappa}\left(m^{2}+n^{2}\right)\left(\frac{\sigma B_{o}^{2}}{\rho}+\frac{v}{k^{*}}\right)-\frac{2}{n j}\left(m^{2}+n^{2}\right)\left(v+\frac{\kappa}{\rho}\right)\right], \\
& a_{4}=\left[\frac{\rho}{n \kappa}\left(U+c_{0}\right)\left(\frac{\sigma B_{o}^{2}}{\rho}+\frac{v}{k^{*}}\right)+\frac{2}{n j}\left(U+c_{0}\right)\right],
\end{aligned}
$$


$a_{5}=\frac{2}{n j}\left(\frac{\sigma B_{o}^{2}}{\rho}+\frac{v}{k^{*}}\right)$ and $a_{6}=\frac{2 m c_{0}}{n j\left(m^{2}+n^{2}\right)}\left(\frac{\sigma B_{o}^{2}}{\rho}+\frac{v}{k^{*}}\right)$.

The solution of Eq. (27) is

$$
u(\xi)=a_{7} e^{r_{11} \xi}+a_{8} e^{r_{12} \xi}+a_{9} e^{r_{13} \xi}+a_{10} e^{r_{14} \xi}+a_{11},
$$

where $r_{11}, r_{12}, r_{13}$, and $r_{14}$ are the roots of the auxiliary equation

$$
a_{1} m^{4}-a_{2} m^{3}+a_{3} m^{2}+a_{4} m+a_{5}=0 .
$$

Putting the Eq. (28) into Eq. (21), we get

$$
v(\xi)=a_{12} e^{r_{11} \xi}+a_{13} e^{r_{12} \xi}+a_{14} e^{r_{13} \xi}+a_{15} e^{r_{14} \xi}+a_{16},
$$

where $a_{i+5}=-\frac{a_{i} m}{n} ; i=7,8,9,10$, and

$$
a_{16}=\frac{1}{n}\left(c_{0}-a_{11} m\right) .
$$

Substituting Eq. (28) into Eq. (26), we get

$$
N(\xi)=a_{17} e^{r_{11} \xi}+a_{18} e^{r_{12} \xi}+a_{19} e^{r_{13} \xi}+a_{20} e^{r_{14} \xi}+a_{21} \xi+a_{22},
$$

where

$$
\begin{gathered}
a_{i}=\frac{1}{r_{i-6}}\left[\frac{a_{i-10} r_{i-6} \rho}{n \kappa}\left(U+c_{0}\right)-\frac{a_{i-10} r_{i-6}^{2} \rho}{n \kappa}\left(m^{2}+n^{2}\right)\left(v+\frac{\kappa}{\rho}\right)\right. \\
\left.+\frac{a_{i-10} \rho}{n \kappa}\left(\frac{\sigma B_{o}^{2}}{\rho}+\frac{v}{k^{*}}\right)\right], i=17,18,19,20,
\end{gathered}
$$

and

$$
a_{21}=\left[\left(\frac{\sigma B_{o}^{2}}{\rho}+\frac{v}{k^{*}}\right)\left(\frac{a_{11} \rho}{n \kappa}-\frac{m \rho c_{0}}{n \kappa\left(m^{2}+n^{2}\right)}\right)\right] .
$$

Putting the Eqs. (28) and (30) into Eq. (22), we get

$$
p(\xi)=a_{23} e^{r_{11} \xi}+a_{24} e^{r_{12} \xi}+a_{25} e^{r_{13} \xi}+a_{26} e^{r_{14} \xi}+a_{27} \xi^{2}+a_{28} \xi+a_{29},
$$

where

$$
\begin{aligned}
a_{i}=\frac{1}{r_{i-12}} & {\left[\frac{a_{i-16} r_{i-12}^{2}}{m}\left(m^{2}+n^{2}\right)\left(v+\frac{\kappa}{\rho}\right)-\frac{a_{i-16} r_{i-12}}{m}\left(U+c_{0}\right)+\frac{a_{i-6} n \kappa}{r_{i-12} \rho}\right.} \\
& \left.-a_{i-16}\left(\frac{\sigma B_{o}^{2}}{\rho}+\frac{v}{k^{*}}\right)\right], i=23,24,25,26,
\end{aligned}
$$

and

$$
a_{27}=\frac{a_{21} n \kappa}{2 \rho}, a_{28}=\frac{a_{22} n \kappa}{\rho} .
$$

Hence, the velocity components, microrotation, and pressure in the original variables form are 


$$
\begin{aligned}
u(x, y, t)= & a_{7} e^{r_{11}(m x+n y+U t)}+a_{8} e^{r_{12}(m x+n y+U t)}+a_{9} e^{r_{13}(m x+n y+U t)} \\
& +a_{10} e^{r_{14}(m x+n y+U t)}+a_{11} \\
v(x, y, t)= & a_{12} e^{r_{11}(m x+n y+U t)}+a_{13} e^{r_{12}(m x+n y+U t)}+a_{14} e^{r_{13}(m x+n y+U t)} \\
& +a_{15} e^{r_{14}(m x+n y+U t)}+a_{16} \\
N(x, y, t)= & a_{17} e^{r_{11}(m x+n y+U t)}+a_{18} e^{r_{12}(m x+n y+U t)}+a_{19} e^{r_{13}(m x+n y+U t)} \\
& +a_{20} e^{r_{14}(m x+n y+U t)}+a_{21}(m x+n y+U t)+a_{22}, \\
p(x, y, t)= & a_{23} e^{r_{11}(m x+n y+U t)}+a_{24} e^{r_{12}(m x+n y+U t)}+a_{25} e^{r_{13}(m x+n y+U t)} \\
& +a_{26} e^{r_{14}(m x+n y+U t)}+a_{27}(m x+n y+U t)^{2}+a_{28}(m x+n y+U t)+a_{29} .
\end{aligned}
$$

\section{Case-II: $U+c_{o}=0$}

For this case, Eqs. (22-24) reduce to

$$
\begin{gathered}
-m p^{\prime}+\left(v+\frac{\kappa}{\rho}\right)\left(m^{2}+n^{2}\right) u^{\prime \prime}+\frac{n \kappa}{\rho} N^{\prime}-\left(\frac{\sigma B_{o}^{2}}{\rho}+\frac{v}{k^{*}}\right) u=0, \\
-n p^{\prime}+\left(v+\frac{\kappa}{\rho}\right)\left(m^{2}+n^{2}\right) v^{\prime \prime}-\frac{m \kappa}{\rho} N^{\prime}-\left(\frac{\sigma B_{o}^{2}}{\rho}+\frac{v}{k^{*}}\right) v=0, \\
\frac{\gamma}{\rho j}\left(m^{2}+n^{2}\right) N^{\prime \prime}-\frac{2 \kappa}{\rho j} N-\frac{\kappa}{\rho j}\left(n u^{\prime}-m v^{\prime}\right)=0 .
\end{gathered}
$$

Eliminating $p$ from Eqs. (36) and (37)

$$
\left(v+\frac{\kappa}{\rho}\right)\left(m^{2}+n^{2}\right)\left(n u^{\prime \prime}-m v^{\prime \prime}\right)+\frac{\kappa}{\rho}\left(m^{2}+n^{2}\right) N^{\prime}-\left(\frac{\sigma B_{o}^{2}}{\rho}+\frac{v}{k^{*}}\right)(n u-m v)=0 .
$$

Converting $v$ into $u$ in Eq. (39), then

$$
N^{\prime}=\frac{\rho}{\kappa}\left[\frac{1}{n}\left(\frac{\sigma B_{o}^{2}}{\rho}+\frac{v}{k^{*}}\right) u-\frac{1}{n}\left(m^{2}+n^{2}\right)\left(v+\frac{\kappa}{\rho}\right) u^{\prime \prime}-\frac{m c_{o}}{n\left(m^{2}+n^{2}\right)}\left(\frac{\sigma B_{o}^{2}}{\rho}+\frac{v}{k^{*}}\right)\right] .
$$

Eliminating $N$ from Eqs. (38) and (40), we get

$$
b_{1} u^{i \mathrm{v}}+b_{2} u^{\prime \prime}+b_{3} u=b_{4} .
$$

where

$$
\begin{aligned}
& b_{1}=\frac{\gamma}{n j \kappa}\left(m^{2}+n^{2}\right)^{2}\left(v+\frac{\kappa}{\rho}\right), \\
& b_{2}=\left[\frac{\kappa}{n \rho j}\left(m^{2}+n^{2}\right)-\frac{\gamma}{n j \kappa}\left(m^{2}+n^{2}\right)\left(\frac{\sigma B_{o}^{2}}{\rho}+\frac{v}{k^{*}}\right)-\frac{2\left(m^{2}+n^{2}\right)}{n j}\left(v+\frac{\kappa}{\rho}\right)\right], \\
& b_{3}=\frac{2}{n j}\left(\frac{\sigma B_{o}^{2}}{\rho}+\frac{v}{k^{*}}\right) \text { and } b_{4}=\frac{2 m c_{o}}{n j\left(m^{2}+n^{2}\right)}\left(\frac{\sigma B_{o}^{2}}{\rho}+\frac{v}{k^{*}}\right) .
\end{aligned}
$$

The solution of Eq.(41) is

$$
u(\xi)=b_{5} e^{r_{21} \xi}+b_{6} e^{r_{22} \xi}+b_{7} e^{r_{23} \xi}+b_{8} e^{r_{24} \xi}+b_{9},
$$

where $r_{21}, r_{22}, r_{23}$, and $r_{24}$ are the roots of the auxiliary equation.

$$
b_{1} m^{4}+b_{2} m^{2}+b_{3}=0 .
$$


Putting Eq. (42) into Eq.(21) provides

$$
v(\xi)=b_{10} e^{r_{21} \xi}+b_{11} e^{r_{22} \xi}+b_{12} e^{r_{23} \xi}+b_{13} e^{r_{24} \xi}+b_{14},
$$

where

$$
\begin{gathered}
b_{i}=-\frac{m b_{i-5}}{n}, \text { for } i=10,11,12,13, \\
b_{14}=\frac{1}{n}\left(c_{0}-m b_{9}\right) .
\end{gathered}
$$

Putting the Eq. (42) in Eq. (40)

$$
N(\xi)=b_{15} e^{r_{21} \xi}+b_{16} e^{r_{22} \xi}+b_{17} e^{r_{23} \xi}+b_{18} e^{r_{24} \xi}+b_{19} \xi+b_{20}
$$

where

$$
b_{i}=\frac{1}{r_{i+6}}\left[\frac{b_{i-10} \rho}{n \kappa}\left(\frac{\sigma B_{o}^{2}}{\rho}+\frac{v}{k^{*}}\right)-\frac{b_{i-10} r_{i+6}^{2} \rho}{n \kappa}\left(m^{2}+n^{2}\right)\left(v+\frac{\kappa}{\rho}\right)\right] \text {, for } i=15,16,17,18,
$$

and

$$
b_{19}=\left(\frac{\sigma B_{o}^{2}}{\rho}+\frac{v}{k^{*}}\right)\left[\frac{b_{9} \rho}{n \kappa}-\frac{m c_{0} \rho}{n \kappa\left(m^{2}+n^{2}\right)}\right] .
$$

Substituting the Eqs. (42) and (44) in Eq. (36), we get

$$
p(\xi)=b_{21} e^{r_{21} \xi}+b_{22} e^{r_{22} \xi}+b_{23} e^{r_{23} \xi}+b_{24} e^{r_{24} \xi}+b_{25} \xi+b_{26},
$$

where

$b_{i}=\frac{1}{r_{i}}\left[\frac{b_{i-16} r_{i}^{2}}{m}\left(m^{2}+n^{2}\right)\left(v+\frac{\kappa}{\rho}\right)+\frac{b_{i-6} n \kappa}{m \rho}-\frac{b_{i-16}}{m}\left(\frac{\sigma B_{o}^{2}}{\rho}+\frac{v}{k^{*}}\right)\right]$, for $i=21,22,23,24$,

and

$$
b_{25}=\frac{b_{19} n \kappa}{m \rho}-\frac{b_{9}}{m}\left(\frac{\sigma B_{o}^{2}}{\rho}+\frac{v}{k^{*}}\right) .
$$

Returning the original variable in Eqs. (42-45), we have

$$
\begin{aligned}
& u(x, y, t)=b_{5} e^{r_{21}(m x+n y+U t)}+b_{6} e^{r_{22}(m x+n y+U t)}+b_{7} e^{r_{23}(m x+n y+U t)} \\
& +b_{8} e^{r_{24}(m x+n y+U t)}+b_{9} \text {, } \\
& v(x, y, t)=b_{10} e^{r_{21}(m x+n y+U t)}+b_{11} e^{r_{22}(m x+n y+U t)}+b_{12} e^{r_{23}(m x+n y+U t)} \\
& +b_{13} e^{r_{24}(m x+n y+U t)}+b_{14} \\
& N(x, y, t)=b_{15} e^{r_{21}(m x+n y+U t)}+b_{16} e^{r_{22}(m x+n y+U t)}+b_{17} e^{r_{23}(m x+n y+U t)} \\
& +b_{18} e^{r_{24}(m x+n y+U t)}+b_{19}(m x+n y+U t)+b_{20}, \\
& p(x, y, t)=b_{21} e^{r_{21}(m x+n y+U t)}+b_{22} e^{r_{22}(m x+n y+U t)}+b_{23} e^{r_{23}(m x+n y+U t)} \\
& +b_{24} e^{r_{24}(m x+n y+U t)}+b_{25}(m x+n y+U t)+b_{26} \text {. }
\end{aligned}
$$


Case - III : $m=0$

In this case, Eqs. (4-7) will be

$$
\begin{aligned}
v^{\prime} & =0, \\
(U+n v) u^{\prime} & =n^{2}\left(v+\frac{\kappa}{\rho}\right) u^{\prime \prime}+\frac{n \kappa}{\rho} N^{\prime}-\left(\frac{\sigma B_{o}^{2}}{\rho}+\frac{v}{k^{*}}\right) u, \\
(U+n v) v^{\prime} & =n^{2}\left(v+\frac{\kappa}{\rho}\right) v^{\prime \prime}-n p^{\prime}-\left(\frac{\sigma B_{o}^{2}}{\rho}+\frac{v}{k^{*}}\right) v, \\
(U+n v) N^{\prime} & =\frac{n^{2} \gamma}{\rho j} N^{\prime \prime}-\frac{2 \kappa}{\rho j} N-\frac{n \kappa}{\rho j} u^{\prime} .
\end{aligned}
$$

From Eq. (51)

$$
N^{\prime}=\frac{\rho}{n \kappa}\left[(U+n v) u^{\prime}-n^{2}\left(v+\frac{\kappa}{\rho}\right) u^{\prime \prime}+\left(\frac{\sigma B_{o}^{2}}{\rho}+\frac{v}{k^{*}}\right) u\right] .
$$

Putting the Eq. (54) in Eq. (53), we find

$$
c_{1} u^{i v}-c_{2} u^{\prime \prime \prime}+c_{3} u^{\prime \prime}+c_{4} u^{\prime}+c_{5} u=0,
$$

where

$$
\begin{aligned}
& c_{1}=\frac{n^{4} \gamma}{n \kappa j}\left(v+\frac{\kappa}{\rho}\right), \\
& c_{2}=\left[\frac{n \rho}{\kappa}(U+n v)\left(v+\frac{\kappa}{\rho}\right)+\frac{n \gamma}{\kappa j}(U+n v)\right], \\
& c_{3}=\left[\frac{\rho}{n \kappa}(U+n v)^{2}+\frac{n \kappa}{\rho j}-\frac{n \gamma}{j \kappa}\left(\frac{\sigma B_{o}^{2}}{\rho}+\frac{v}{k^{*}}\right)-\frac{2 n}{j}\left(v+\frac{\kappa}{\rho}\right)\right], \\
& c_{4}=\left[\frac{\rho}{n \kappa}(U+n v)\left(\frac{\sigma B_{o}^{2}}{\rho}+\frac{v}{k^{*}}\right)+\frac{2 n}{j}(U+n v)\right], \\
& c_{5}=\frac{2}{n j}\left(\frac{\sigma B_{o}^{2}}{\rho}+\frac{v}{k^{*}}\right) .
\end{aligned}
$$

The solution of Eq.(55) is

$$
u(\xi)=c_{6} e^{r_{31} \xi}+c_{7} e^{r_{32} \xi}+c_{8} e^{r_{33} \xi}+c_{9} e^{r_{34} \xi},
$$

where $r_{31}, r_{32}, r_{33}$, and $r_{34}$ are the roots of the auxiliary equation.

$$
c_{1} m^{4}-c_{2} m^{3}+c_{3} m^{2}+c_{4} m+c_{5}=0 .
$$

Integrating Eq. (50), we have

$$
v(\xi)=c_{10} .
$$

Putting the Eq. (56) in Eq. (54)

$$
N(\xi)=c_{11} e^{r_{31} \xi}+c_{12} e^{r_{32} \xi}+c_{13} e^{r_{33} \xi}+c_{14} e^{r_{34} \xi}+c_{15} .
$$


where

$c_{i}=\frac{1}{r_{i+20}}\left[\frac{c_{i-5} r_{i+20} \rho}{n \kappa}(U+n v)-\frac{c_{i-5} r_{i+20}^{2} m^{2} \rho}{n \kappa}\left(v+\frac{\kappa}{\rho}\right)+c_{i-5}\left(\frac{\sigma B_{o}^{2}}{\rho}+\frac{v}{k^{*}}\right)\right]$, for $i=11,12,13,14$.

Putting Eqs. (50) and (57) into Eq. (52)

$$
p(\xi)=c_{16} \xi+c_{17}
$$

where

$$
c_{16}=-\frac{c_{10}}{n}\left(\frac{\sigma B_{o}^{2}}{\rho}+\frac{v}{k^{*}}\right) .
$$

Returning the original variable, we get

$$
\begin{aligned}
u(x, y, t)= & c_{6} e^{r_{31}(m x+n y+U t)}+c_{7} e^{r_{32}(m x+n y+U t)}+c_{8} e^{r_{33}(m x+n y+U t)} \\
& +c_{9} e^{r_{34}(m x+n y+U t)}, \\
v(x, y, t)= & c_{10}, \\
N(x, y, t)= & c_{11} e^{r_{31}(m x+n y+U t)}+c_{12} e^{r_{32}(m x+n y+U t)}+c_{13} e^{r_{33}(m x+n y+U t)} \\
& +c_{14} e^{r_{34}(m x+n y+U t)}+c_{15}, \\
p(x, y, t)= & c_{16}(m x+n y+U t)+c_{17} .
\end{aligned}
$$

\section{Case- IV: $\mathbf{n}=\mathbf{0}$}

Equations $(4-7)$ reduces to

$$
u^{\prime}=0
$$

$$
\begin{aligned}
& (U+m u) u^{\prime}=-m p^{\prime}+m^{2}\left(v+\frac{\kappa}{\rho}\right) u^{\prime \prime}-\left(\frac{\sigma B_{o}^{2}}{\rho}+\frac{v}{k^{*}}\right) u, \\
& (U+m u) v^{\prime}=m^{2}\left(v+\frac{\kappa}{\rho}\right) v^{\prime \prime}-\frac{m \kappa}{\rho} N^{\prime}-\left(\frac{\sigma B_{o}^{2}}{\rho}+\frac{v}{k^{*}}\right) v, \\
& (U+m u) N^{\prime}=\frac{m^{2} \gamma}{\rho j} N^{\prime \prime}-\frac{2 \kappa}{\rho j} N+m v^{\prime} .
\end{aligned}
$$

Integrating Eq. (64), we get

$$
u(\xi)=d_{1}
$$

From Eq. (66), we have

$$
N^{\prime}=\frac{\rho}{m \kappa}\left[m\left(v+\frac{\kappa}{\rho}\right) v^{\prime \prime}-\frac{1}{m}\left(U+d_{1} m\right) v^{\prime}-\frac{1}{m}\left(\frac{\sigma B_{o}^{2}}{\rho}+\frac{v}{k^{*}}\right) v\right] .
$$

Putting Eq. (69) into Eq. (67)

$$
d_{2} v^{i \mathrm{v}}-d_{3} v^{\prime \prime \prime}-d_{4} v^{\prime \prime}+d_{5} v^{\prime}+d_{6} v=0
$$


where

$$
\begin{gathered}
d_{2}=\frac{m^{3} \gamma}{\kappa j}\left(v+\frac{k}{\rho}\right), \\
d_{3}=\left[\frac{m \gamma}{\kappa j}\left(U+d_{1} m\right)-\frac{m \rho}{\kappa}\left(U+d_{1} m\right)\left(v+\frac{\kappa}{\rho}\right)\right], \\
d_{4}=\left[\frac{m \gamma}{\kappa j}\left(\frac{\sigma B_{o}^{2}}{\rho}+\frac{v}{k^{*}}\right)-\frac{\rho}{m \kappa}\left(U+d_{1} m\right)^{2}+\frac{2 m}{j}\left(v+\frac{\kappa}{\rho}\right)-m\right], \\
d_{5}=\left[\frac{2}{m j}\left(U+d_{1} m\right)+\frac{\rho}{m \kappa}\left(U+d_{1} m\right)\left(\frac{\sigma B_{o}^{2}}{\rho}+\frac{v}{k^{*}}\right)\right], \\
d_{6}=\frac{2}{m j}\left(\frac{\sigma B_{o}^{2}}{\rho}+\frac{v}{k^{*}}\right) .
\end{gathered}
$$

The solution of Eq. (70) is

$$
v(\xi)=d_{7} e^{r_{41} \xi}+d_{8} e^{r_{42} \xi}+d_{9} e^{r_{43} \xi}+d_{10} e^{r_{44} \xi},
$$

where $r_{41}, r_{42}, r_{43}$, and $r_{44}$ are the roots of the auxiliary equation

$$
d_{2} m^{4}-d_{3} m^{3}-d_{4} m^{2}+d_{5} m+d_{6}=0 .
$$

Putting the Eq. (71) in Eq. (69)

$$
N(\xi)=d_{11} e^{r_{41} \xi}+d_{12} e^{r_{42} \xi}+d_{13} e^{r_{43} \xi}+d_{14} e^{r_{44} \xi}+d_{15},
$$

where

$d_{i}=\frac{1}{r_{i+30}}\left[\frac{d_{i-4} r_{i+30}^{2} m \rho}{\kappa}\left(v+\frac{\kappa}{\rho}\right)-\frac{d_{i-4} r_{i+30} \rho}{m \kappa}\left(U+d_{1} m\right)-\frac{d_{i-4} \rho}{m \kappa}\left(\frac{\sigma B_{o}^{2}}{\rho}+\frac{v}{k^{*}}\right)\right]$. for $i=11,12,13,14$,

Substituting the Eq. (68) in Eq. (65)

$$
p(\xi)=d_{16} \xi+d_{17},
$$

where

$$
d_{16}=-\frac{d_{1}}{m}\left(\frac{\sigma B_{o}^{2}}{\rho}+\frac{v}{k^{*}}\right) .
$$

The exact solutions in term of the original variables are

$$
\begin{aligned}
u(x, y, t)= & d_{1}, \\
v(x, y, t)= & d_{7} e^{r_{41}(m x+n y+U t)}+d_{8} e^{r_{42}(m x+n y+U t)}+d_{9} e^{r_{43}(m x+n y+U t)} \\
& +d_{10} e^{r_{44}(m x+n y+U t)} \\
N(x, y, t)= & d_{11} e^{r_{41}(m x+n y+U t)}+d_{12} e^{r_{42}(m x+n y+U t)}+d_{13} e^{r_{43}(m x+n y+U t)} \\
& +d_{14} e^{r_{44}(m x+n y+U t)}+d_{15}, \\
p(x, y, t)= & d_{16}(m x+n y+U t)+d_{17} .
\end{aligned}
$$


Jami et al. Journal of the Egyptian Mathematical Society

(2020) $28: 23$

Page 12 of 22

Case-V: $U=0$

For this case, Eqs. (22)-(24) become

$$
\begin{aligned}
& c_{0} u^{\prime}=-m p^{\prime}+\left(m^{2}+n^{2}\right)\left(v+\frac{\kappa}{\rho}\right) u^{\prime \prime}+\frac{n \kappa}{\rho} N^{\prime}-\left(\frac{\sigma B_{o}^{2}}{\rho}+\frac{v}{k^{*}}\right) u, \\
& c_{0} v^{\prime}=-n p^{\prime}+\left(m^{2}+n^{2}\right)\left(v+\frac{\kappa}{\rho}\right) v^{\prime \prime}-\frac{m \kappa}{\rho} N^{\prime}-\left(\frac{\sigma B_{o}^{2}}{\rho}+\frac{v}{k^{*}}\right) v, \\
& c_{0} N^{\prime}=\frac{\gamma}{\rho j}\left(m^{2}+n^{2}\right) N^{\prime \prime}-\frac{2 \kappa}{\rho j} N-\frac{\kappa}{\rho j}\left(n u^{\prime}-m v^{\prime}\right) .
\end{aligned}
$$

Eliminating $p$ from Eqs. (78) and (79), we get

$$
\begin{aligned}
c_{0}\left(n u^{\prime}-m v^{\prime}\right)= & \left(m^{2}+n^{2}\right)\left(v+\frac{\kappa}{\rho}\right)\left(n u^{\prime \prime}-m v^{\prime \prime}\right)+\frac{\kappa}{\rho}\left(m^{2}+n^{2}\right) N^{\prime} \\
& -\left(\frac{\sigma B_{o}^{2}}{\rho}+\frac{v}{k^{*}}\right)(n u-m v) .
\end{aligned}
$$

Convert ing $v$ into $u$ in Eq. (81), we arrive

$$
\begin{aligned}
\frac{c_{0}}{n}\left(m^{2}+n^{2}\right) u^{\prime}= & \frac{1}{n}\left(m^{2}+n^{2}\right)^{2}\left(v+\frac{\kappa}{\rho}\right) u^{\prime \prime}+\frac{\kappa}{\rho}\left(m^{2}+n^{2}\right) N^{\prime} \\
& -\frac{\left(m^{2}+n^{2}\right)}{n}\left(\frac{\sigma B_{o}^{2}}{\rho}+\frac{v}{k^{*}}\right) u \\
& +\frac{m c_{0}}{n}\left(\frac{\sigma B_{o}^{2}}{\rho}+\frac{v}{k^{*}}\right) .
\end{aligned}
$$

From Eq. (82), we have

$$
N^{\prime}=\frac{\rho}{\kappa}\left[\frac{c_{0}}{n} u^{\prime}-\frac{1}{n}\left(m^{2}+n^{2}\right)\left(v+\frac{\kappa}{\rho}\right) u^{\prime \prime}+\frac{1}{n}\left(\frac{\sigma B_{o}^{2}}{\rho}+\frac{v}{k^{*}}\right) u-\frac{m c_{0}}{n\left(m^{2}+n^{2}\right)}\left(\frac{\sigma B_{o}^{2}}{\rho}+\frac{v}{k^{*}}\right)\right] .
$$

Putting Eq. (83) in Eq. (78)

$$
e_{1} u^{i \mathrm{v}}-e_{2} u^{\prime \prime \prime}+e_{3} u^{\prime \prime}+e_{4} u^{\prime}+e_{5} u=e_{6},
$$

where

$$
\begin{aligned}
& e_{1}=\frac{\gamma\left(m^{2}+n^{2}\right)^{2}}{n \kappa j}\left(v+\frac{\kappa}{\rho}\right), \\
& e_{2}=\left[\frac{c_{0} \rho}{n \kappa}\left(m^{2}+n^{2}\right)\left(v+\frac{\kappa}{\rho}\right)+\frac{c_{0} \gamma}{n j \kappa}\left(m^{2}+n^{2}\right)\right], \\
& e_{3}=\left[\frac{\rho c_{0}^{2}}{n \kappa}-\frac{\gamma\left(m^{2}+n^{2}\right)}{n j \kappa}\left(\frac{\sigma B_{o}^{2}}{\rho}+\frac{v}{k^{*}}\right)-\frac{2\left(m^{2}+n^{2}\right)}{n j}\left(v+\frac{\kappa}{\rho}\right)+\frac{\kappa}{\rho j}\left(m^{2}+n^{2}\right)\right], \\
& e_{4}= {\left[\frac{\rho c_{o}}{n \kappa}\left(\frac{\sigma B_{o}^{2}}{\rho}+\frac{v}{k^{*}}\right)+\frac{2 c_{o}}{n j}\right], } \\
& e_{5}=\frac{2}{n j}\left(\frac{\sigma B_{o}^{2}}{\rho}+\frac{v}{k^{*}}\right) \text { and } e_{6}=\frac{2 m c_{o}}{n\left(m^{2}+n^{2}\right)}\left(\frac{\sigma B_{o}^{2}}{\rho}+\frac{v}{k^{*}}\right) .
\end{aligned}
$$


The solution of Eq. (84) is

$$
u(\xi)=e_{7} e^{r_{51} \xi}+e_{8} e^{r_{52} \xi}+e_{9} e^{r_{53} \xi}+e_{10} e^{r_{54} \xi}+e_{11},
$$

where $r_{51}, r_{52}, r_{53}$, and $r_{54}$ are the roots of the auxiliary equation

$$
e_{1} m^{4}-e_{2} m^{3}+e_{3} m^{2}+e_{4} m+e_{5}=0 .
$$

Substituting Eq. (85) into Eq. (21)

$$
v(\xi)=e_{12} e^{r_{51} \xi}+e_{13} e^{r_{52} \xi}+e_{14} e^{r_{53} \xi}+e_{15} e^{r_{54} \xi}+e_{16},
$$

where

$$
\begin{gathered}
e_{i}=-\frac{m e_{i-5}}{n}, \text { for } i=12,13,14,15, \text { and } \\
e_{16}=\frac{1}{n}\left(c_{0}-m e_{11}\right) .
\end{gathered}
$$

Putting the Eq. (85) in Eq. (83)

$$
N(\xi)=e_{17} e^{r_{51} \xi}+e_{18} e^{r_{52} \xi}+e_{19} e^{r_{53} \xi}+e_{20} e^{r_{54} \xi}+e_{21} \xi+e_{22} .
$$

where

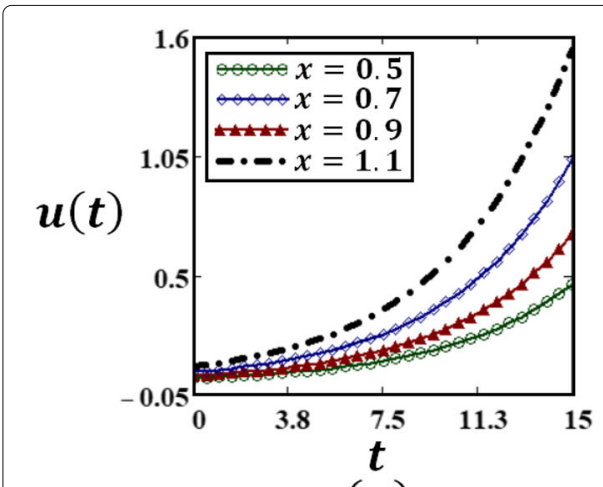

(a)

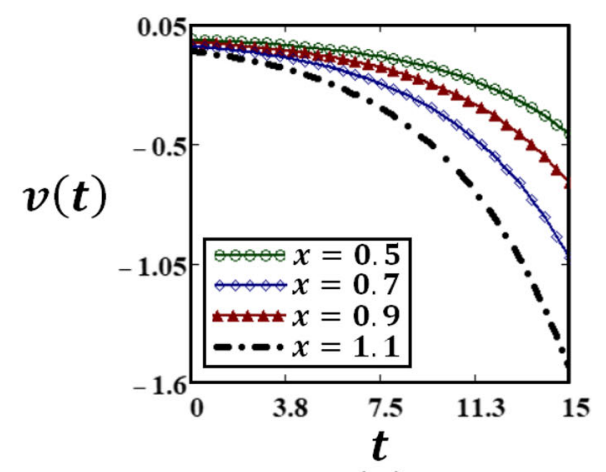

(b)

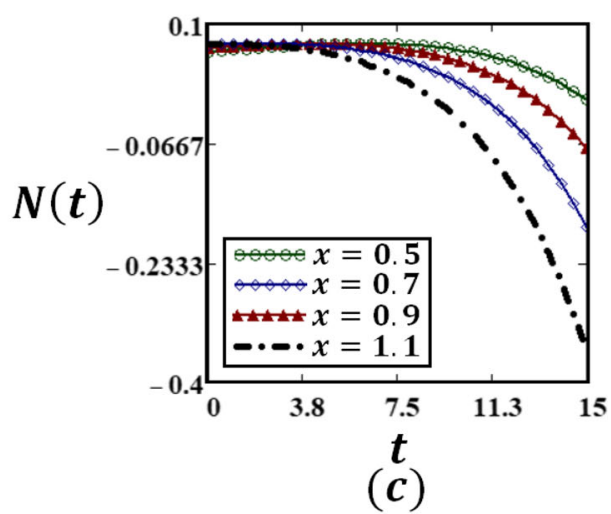

Fig. 2 Profiles of the velocity field $u(x, t), v(x, t)$, and microrotation $N(x, t)$ for MHD micropolar fluid given by Eqs. (32), (33), and (34), for $U=0.1, \quad v=0.045, \mu=0.5, \kappa=0.2, \quad B_{0}=2, k^{*}=0.2, \sigma=0.2, j=$ $0.03, m=n=1, y=1, c_{0}=0.01$, and different values of $x$ 


$$
\begin{aligned}
e_{i}=\frac{1}{r_{i+34}}\left[\frac{e_{i-10} r_{i+34} \rho c_{0}}{n \kappa}-\frac{e_{i-10} r_{i+34}^{2} \rho}{n \kappa}\left(m^{2}+n^{2}\right)\right. \\
\left.\quad \times\left(v+\frac{\kappa}{\rho}\right)+\frac{e_{i-10} \rho}{n \kappa}\left(\frac{\sigma B_{o}^{2}}{\rho}+\frac{v}{k^{*}}\right)\right], \text { for } i=17,18,19,20 \text { and } \\
e_{21}=-\frac{\rho m c_{o}}{n \kappa\left(m^{2}+n^{2}\right)}\left(\frac{\sigma B_{o}^{2}}{\rho}+\frac{v}{k^{*}}\right) .
\end{aligned}
$$

Substituting Eqs. (85) and (87) in Eq. (78), we get

$$
p(\xi)=e_{23} e^{r_{51} \xi}+e_{24} e^{r_{52} \xi}+e_{25} e^{r_{53} \xi}+e_{26} e^{r_{54} \xi}+e_{27} \xi^{2}+e_{28} \xi+e_{29}
$$

where

$$
\begin{gathered}
e_{i}=\frac{1}{r_{i+28}}\left[\frac{e_{i-16} r_{i+28}^{2}}{m}\left(m^{2}+n^{2}\right)\left(v+\frac{\kappa}{\rho}\right)-\frac{e_{i-16} r_{i+28} c_{0}}{m}+\frac{e_{i-6} n \kappa}{\rho}\right. \\
\left.-e_{i-16}\left(\frac{\sigma B_{o}^{2}}{\rho}+\frac{v}{k^{*}}\right)\right], \text { for } i=23,24,25,26, \text { and } \\
e_{27}=\frac{e_{21} n \kappa}{2 \rho} \text { and } e_{28}=-e_{11}\left(\frac{\sigma B_{o}^{2}}{\rho}+\frac{v}{k^{*}}\right) .
\end{gathered}
$$

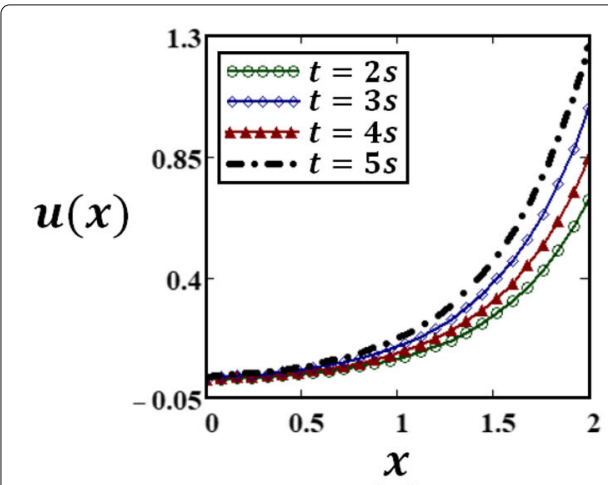

(a)

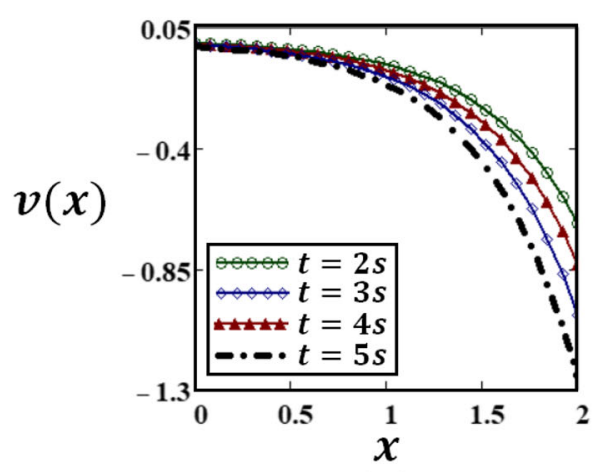

(b)

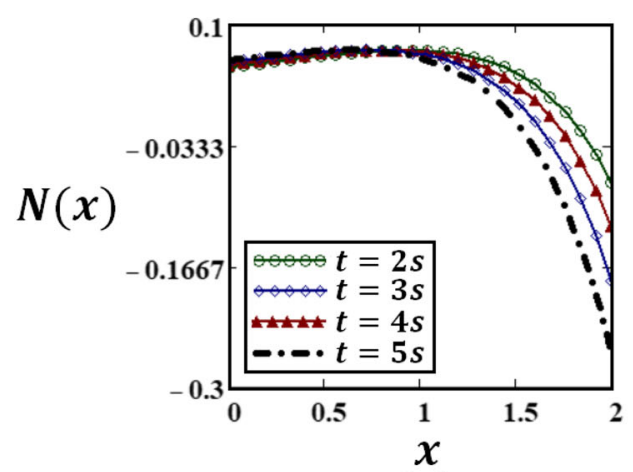

(c)

Fig. 3 Profiles of the velocity field $u(x, t), v(x, t)$ and microrotation $N(x, t)$ for MHD micropolar fluid given by Eqs. (32), (33), and (34), for $U=0.1, \quad v=0.045, \mu=0.5, \quad \kappa=0.2, \quad B_{0}=2, k^{*}=0.2, \sigma=0.2, j=$ $0.03, m=n=1, y=1, c_{0}=0.01$, and different values of $t$ 
Returning to the original variables, the exact solutions are

$$
\begin{aligned}
u(x, y, t)= & e_{7} e^{r_{51}(m x+n y+U t)}+e_{8} e^{r_{52}(m x+n y+U t)}+e_{9} e^{r_{53}(m x+n y+U t)} \\
& +e_{10} e^{r_{54}(m x+n y+U t)}+e_{11} \\
v(x, y, t)= & e_{12} e^{r_{51}(m x+n y+U t)}+e_{13} e^{r_{52}(m x+n y+U t)}+e_{14} e^{r_{53}(m x+n y+U t)} \\
& +e_{15} e^{r_{54}(m x+n y+U t)}+e_{16} \\
N(x, y, t)= & e_{17} e^{r_{51}(m x+n y+U t)}+e_{18} e^{r_{52}(m x+n y+U t)}+e_{19} e^{r_{53}(m x+n y+U t)} \\
& +e_{20} e^{r_{54}(m x+n y+U t)}+e_{21}(m x+n y+U t)+e_{22}, \\
p(x, y, t)= & e_{23} e^{r_{51}(m x+n y+U t)}+e_{24} e^{r_{52}(m x+n y+U t)}+e_{25} e^{r_{53}(m x+n y+U t)} \\
& +e_{26} e^{r_{54}(m x+n y+U t)} e_{27}(m x+n y+U t)^{2}+e_{28}(m x+n y+U t)+e_{29} .
\end{aligned}
$$

\section{Making initial and boundary conditions}

Some exact traveling solutions are determined for Eqs. (4)-(7) in the "Traveling wave solutions" section, without initial and boundary conditions. Definitely, the initial and boundary conditions can be made from these solutions. For instance, if governing Eqs. (4)-(7) is assumed that they are satisfied Rayleigh-Stokes problem for an edge, then for two perpendicular plates ( $y z$ and $x z$-planes), we have $x, y \in[0, \infty]$. Utilizing $t=0, x=0$ and $y=0$ into Eqs. (32)-(35), then some initial and boundary conditions are achieved.

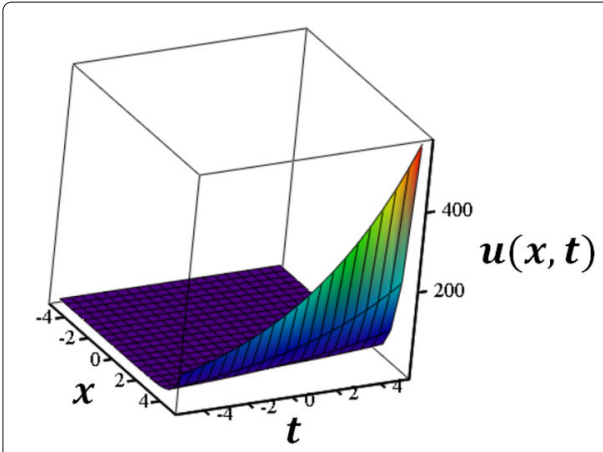

(a)

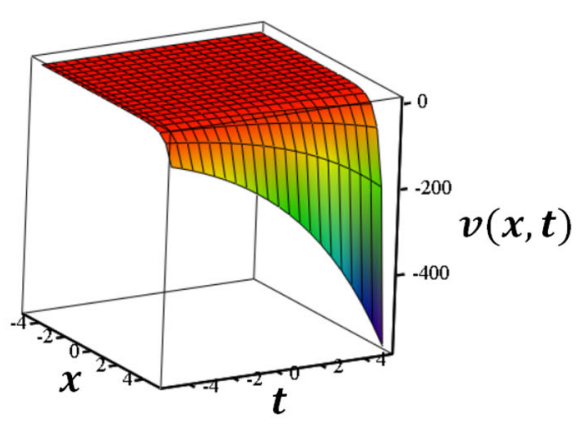

(b)

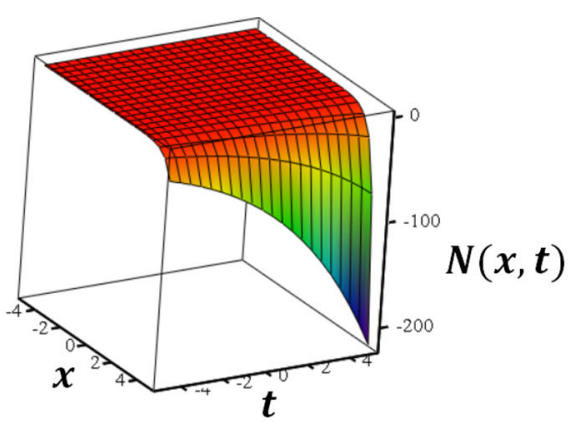

(c)

Fig. 4 3D profiles of the velocity field $u(x, t), v(x, t)$, and microrotation $N(x, t)$ for MHD micropolar fluid given by Eqs. (32), (33), and (34), for $U=0.1, \quad v=0.045, \quad \mu=0.5, \quad \kappa=0.2, \quad B_{0}=2, k^{*}=0.2, \quad \sigma=0.2, j=$ $0.03, m=n=1, y=1, c_{0}=0.01$ 
- Equations (32)-(35) satisfy the following initial conditions:

$$
\begin{aligned}
u(x, y, 0)= & a_{7} e^{r_{11}(m x+n y)}+a_{8} e^{r_{12}(m x+n y)}+a_{9} e^{r_{13}(m x+n y)}+a_{10} e^{r_{14}(m x+n y)} \\
& +a_{11}, x, y>0, \\
v(x, y, 0)= & a_{12} e^{r_{11}(m x+n y)}+a_{13} e^{r_{12}(m x+n y)}+a_{14} e^{r_{13}(m x+n y)}+a_{15} e^{r_{14}(m x+n y)} \\
& +a_{16}, x, y>0, \\
N(x, y, 0)= & a_{17} e^{r_{11}(m x+n y)}+a_{18} e^{r_{12}(m x+n y)}+a_{19} e^{r_{13}(m x+n y)}+a_{20} e^{r_{14}(m x+n y)} \\
& +a_{21}(m x+n y)+a_{22}, x, y>0, \\
& \\
p(x, y, 0)= & a_{23} e^{r_{11}(m x+n y)}+a_{24} e^{r_{12}(m x+n y)}+a_{25} e^{r_{13}(m x+n y)}+a_{26} e^{r_{14}(m x+n y)} \\
& +a_{27}(m x+n y)^{2}+a_{28}(m x+n y)+a_{29}, x, y>0 .
\end{aligned}
$$

- Equations (32)-(35) satisfy the following boundary conditions on the upper plate (yz-plane):

$$
\begin{aligned}
u(0, y, t)= & a_{7} e^{r_{11}(n y+U t)}+a_{8} e^{r_{12}(n y+U t)}+a_{9} e^{r_{13}(n y+U t)}+a_{10} e^{r_{14}(n y+U t)} \\
& +a_{11}, t>0
\end{aligned}
$$
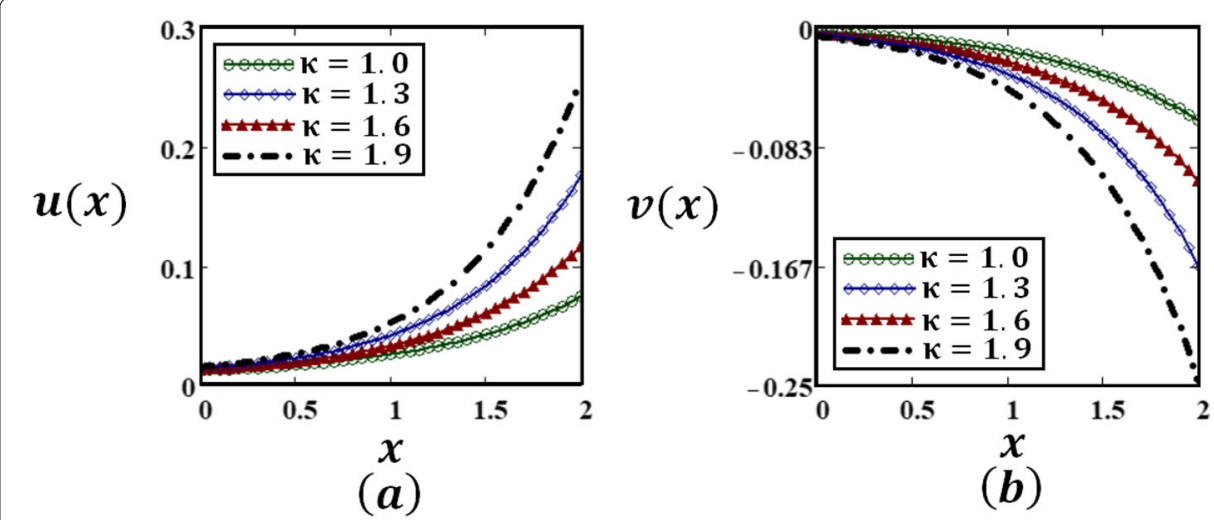

(a)

(b)

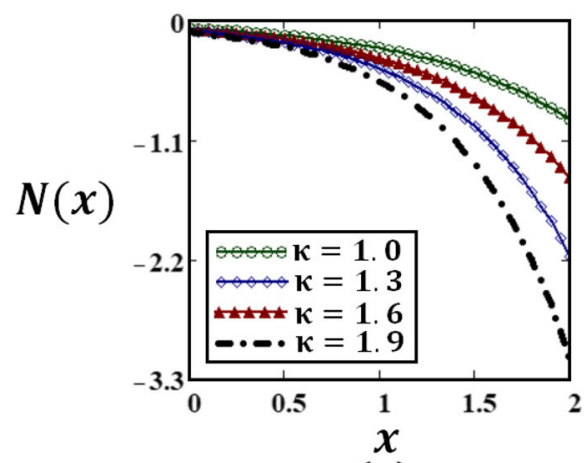

(c)

Fig. 5 Profiles of the velocity field $u(x, t), v(x, t)$, and microrotation $N(x, t)$ for MHD micropolar fluid given by Eqs. (32), (33), and (34), for $U=0.1, \quad v=0.045, \quad \mu=0.5, \quad B_{0}=2, k^{*}=0.2, \quad \sigma=0.2, j=0.03, m=n=$ $1, y=1, c_{0}=0.01, t=2 s$, and different values of $\kappa$ 


$$
\begin{aligned}
\begin{aligned}
v(0, y, t)= & a_{12} e^{r_{11}(n y+U t)}+a_{13} e^{r_{12}(n y+U t)}+a_{14} e^{r_{13}(n y+U t)}+a_{15} e^{r_{14}(n y+U t)} \\
& +a_{16}, \quad t>0, \\
N(0, y, t)= & a_{17} e^{r_{11}(n y+U t)}+a_{18} e^{r_{12}(n y+U t)}+a_{19} e^{r_{13}(n y+U t)}+a_{20} e^{r_{14}(n y+U t)} \\
& +a_{21}(n y+U t)+a_{22}, t>0, \\
p(0, y, t)= & a_{23} e^{r_{11}(n y+U t)}+a_{24} e^{r_{12}(n y+U t)}+a_{25} e^{r_{13}(n y+U t)}+a_{26} e^{r_{14}(n y+U t)} \\
& \quad+a_{27}(n y+U t)^{2}+a_{28}(n y+U t)+a_{29}, t>0 .
\end{aligned}
\end{aligned}
$$

- Equations (32)-(35) satisfy the following boundary conditions on the lower plate (xz-plane):

$$
\begin{aligned}
u(x, 0, t)= & a_{7} e^{r_{11}(m x+U t)}+a_{8} e^{r_{12}(m x+U t)}+a_{9} e^{r_{13}(m x+U t)}+a_{10} e^{r_{14}(m x+U t)} \\
& +a_{11}, \quad t>0 \\
v(x, 0, t)= & a_{12} e^{r_{11}(m x+U t)}+a_{13} e^{r_{12}(m x+U t)}+a_{14} e^{r_{13}(m x+U t)}+a_{15} e^{r_{14}(m x+U t)} \\
& +a_{16}, \quad t>0
\end{aligned}
$$

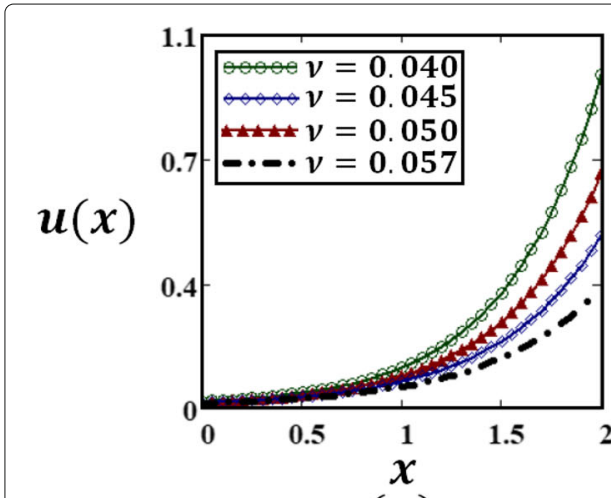

(a)

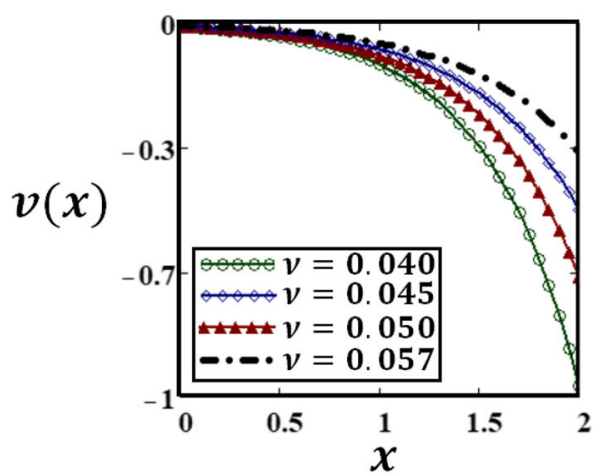

(b)

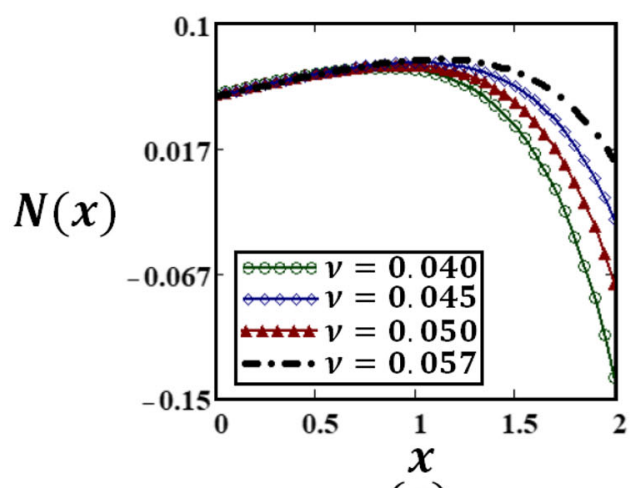

(c)

Fig. 6 Profiles of the velocity field $u(x, t), v(x, t)$, and microrotation $N(x, t)$ for MHD micropolar fluid given by Eqs. (32), (33), and (34), for $U=0.1, \quad \rho=12, \quad \kappa=0.2, \quad B_{0}=2, k^{*}=0.2, \quad \sigma=0.2, j=0.03, m=n=1$, $y=1, c_{0}=0.01, t=2 s$, and different values of $v$ 


$$
\begin{aligned}
N(x, 0, t)= & a_{17} e^{r_{11}(m x+U t)}+a_{18} e^{r_{12}(m x+U t)}+a_{19} e^{r_{13}(m x+U t)}+a_{20} e^{r_{14}(m x+U t)} \\
& +a_{21}(m x+U t)+a_{22}, \quad t>0, \\
p(x, 0, t)= & a_{23} e^{r_{11}(m x+U t)}+a_{24} e^{r_{12}(m x+U t)}+a_{25} e^{r_{13}(m x+U t)}+a_{26} e^{r_{14}(m x+U t)} \\
& +a_{27}(m x+U t)^{2}+a_{28}(m x+U t)+a_{29}, \quad t>0 .
\end{aligned}
$$

Similarly, we can made the initial and boundary conditions to the other many cases.

\section{Numerical results and discussions}

In this paper, we obtain exact traveling wave solutions of MHD flow of micropolar fluid in a porous medium. The methodology in the recent work is easily reduced to the non-linear partial differential equations of MHD micropolar fluid to linearized ordinary differential equations. The method has been used directly without any restrictive assumption and laborious calculation. These exact traveling solutions are obtained for five different cases. From these solutions, we can find special solutions for with and without MHD and porous effects. It is also noted that when $\kappa, N \longrightarrow 0$, we obtain the solutions for viscous fluid flow. Furthermore, in all cases, we write general solutions in the exponential form; however, we can also write general solutions in the oscillatory form by assuming some suitable restriction on the roots of the equations, but we avoid it due to the length of the paper.

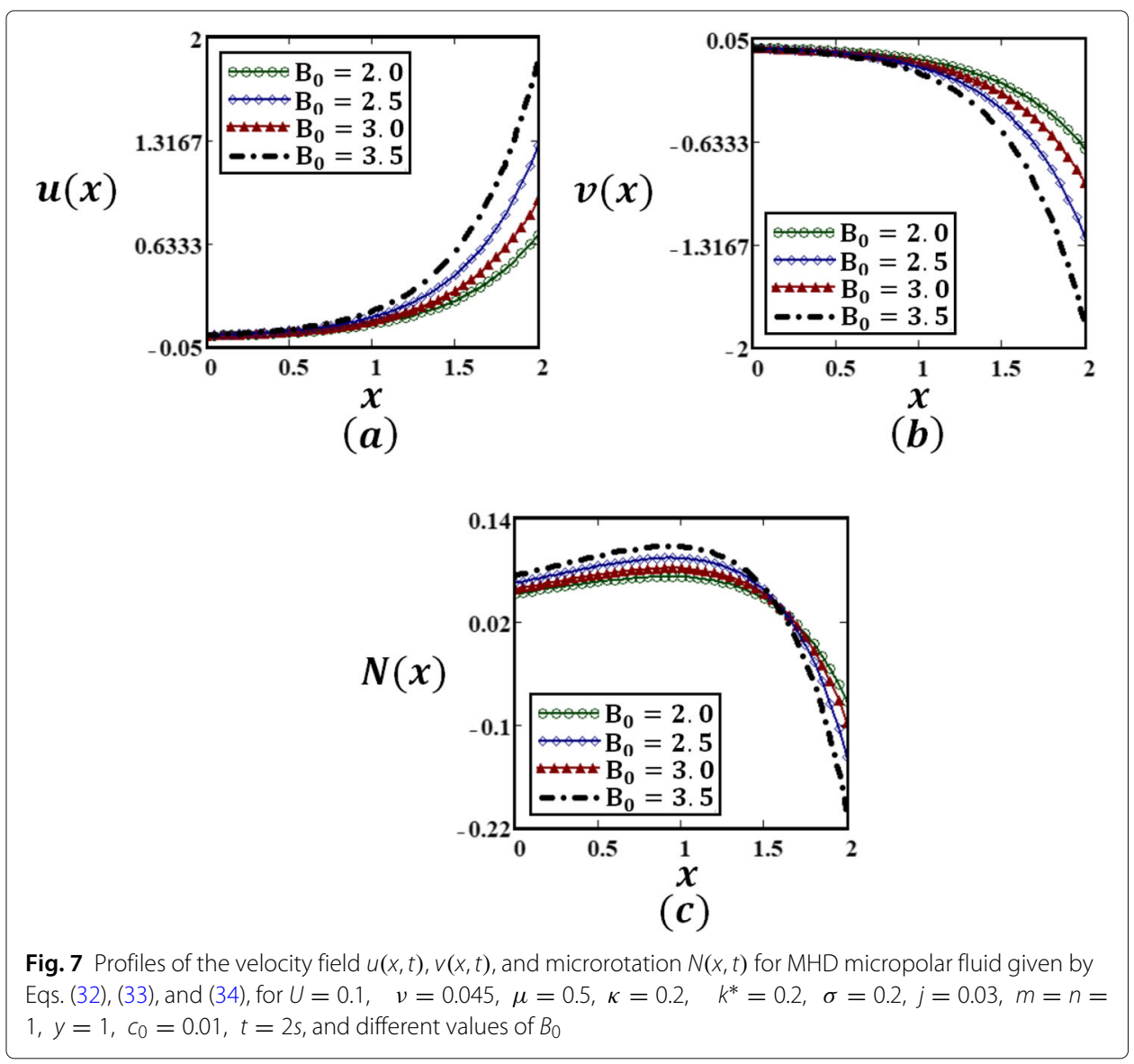


Now, in order to reveal some relevant physical aspect of the determine results the diagram of the velocity fluid components $u(x, t), v(x, t)$, and the microrotation $N(x, t)$ are present against $x$ for different values of $t$ and of the important parameters of the micropolar fluid. For the sake of convenience, we present a graph only for case I, $U+c_{0} \neq 0$, and a similar prediction can be done by other cases. Figures 2, 3, and 4 showed the influence of time, space variable $x$, and three-dimensional representation of $u, v$, and $N$. From Figs. 2 and 3, it is clear that $u$ is increasing function of time $t$ and space variable $x$; however, $v$ and $N$ are also increasing functions (in absolute value) of these variables. The combined effects of these variables are shown in 3D graphs of Fig. 4. It is more clear from 3D pictures of $u, v$, and $N$ that they are becoming strengthen with the strengthening values of $t$ and $x$. The influence of the vortex viscosity $\kappa$ is shown in Fig. 5; from these figures, we can see that velocity components $u, v$, and microrotation $N$ (in absolute sense) increase with the increasing values of $\kappa$. The influence of kinematic viscosity $v$ is shown in Fig. 6. The effect of kinematic viscosity $v$ on a fluid motion is quite opposite to that of vortex viscosity $\kappa$. The effect of magnetic parameter $B_{0}$ is shown in Fig. 7. It is clear that the effect of the magnetic parameter $B_{0}$ is similar to $u$ and $v$. For instance, the velocity component $u$ is increasing while $v$ is also increasing function (in absolute value) of $B_{0}$ because the particle of micropolar fluid always travel rotationally, which may cause increment in velocity.

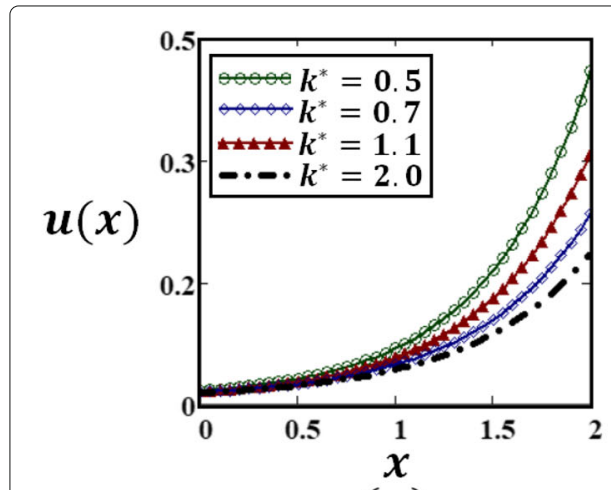

(a)

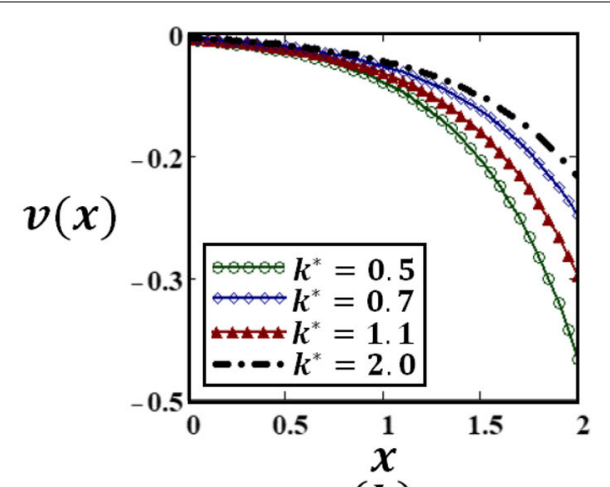

(b)

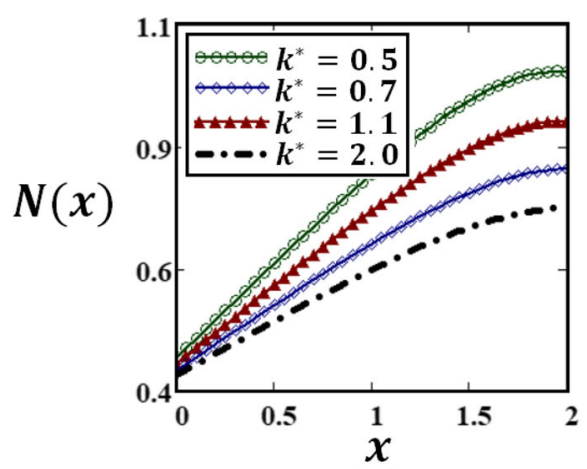

$(c)$

Fig. 8 Profiles of the velocity field $u(x, t), v(x, t)$, and microrotation $N(x, t)$ for MHD micropolar fluid given by Eqs. (32), (33), and (34), for $U=0.1, \quad v=0.045, \mu=0.5, \quad \kappa=0.2, \quad B_{0}=2, \quad \sigma=0.2, j=0.03, m=n=$ $1, y=1, c_{0}=0.01, t=2 s$, and different values of $k^{*}$ 
However, the influence of magnetic parameter $B_{0}$ on the microrotation profile $N$ is decreasing in the two-third domain and increasing in the remaining domain. In Fig. 8, the effect of porosity parameter $k^{*}$ is depicted on the velocity components and microrotation profiles. It is noted that the porosity parameter $k$ has opposite effects on the profiles of $u$ and $v$ in comparison to the magnetic parameter $B_{0}$. The microrotation $N$ is decreasing the function of the porosity parameter. The influence of the parameter $m$ appears in the traveling wave parameter $\xi$ is shown in Fig. 9. It comes to the notice that increasing values of the parameter $m$ reduce the values of $u$ and $N$; however, the values of $v$ are increased very shortly.

Finally for comparison, the profile of the velocity components $u(x, t)$ and $v(x, t)$ corresponding to the motion of the three types of fluids (MHD micropolar fluid in porous medium, micropolar, and MHD Newtonian in porous medium fluids) are together presented in Fig. 10 for similar values of material constants. It is clear from these figures that MHD Newtonian in a porous medium fluid is the fastest, and the simple micropolar fluid is slowest. It is also brought to the knowledge that magnetic and porosity parameters fasten the fluid motion in this case that we have considered here. SI units are used in making all these graphs and Mathcad software is used for making these graphs.

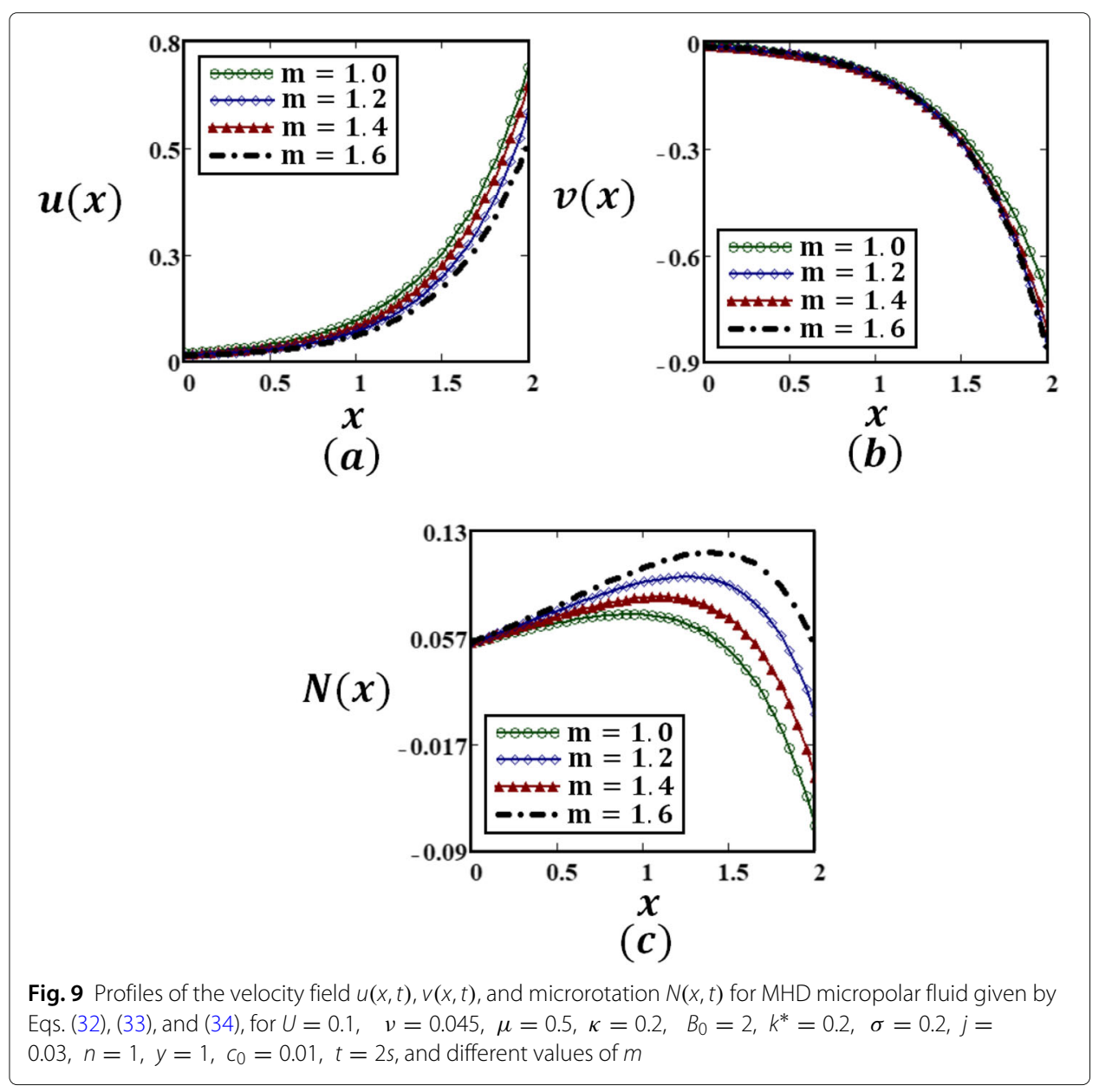



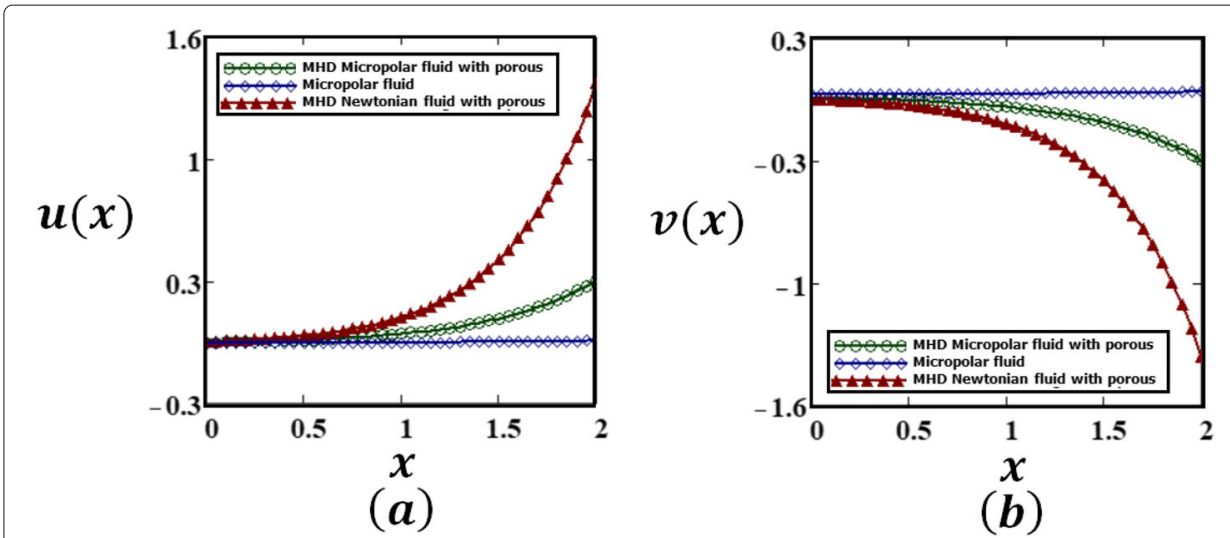

Fig. 10 Profiles of the velocity field $u(x, t)$ and $v(x, t)$ for MHD micropolar fluid with porous effect, micropolar fluid, and MHD Newtonian fluids with porous given by Eqs. (32), (33), and (34), for $U=0.1, \quad v=0.045, \mu=$ $0.5, \kappa=0.2, \quad B_{0}=0.2, k^{*}=0.2, \quad \sigma=0.2, j=0.03, \quad m=n=1, y=1, c_{0}=0.01$, and $t=2 \mathrm{~s}$

\section{Acknowledgements}

The authors would like to express their sincere thanks and gratitude to the referees for their careful assessment and fruitful remarks and suggestions regarding the initial version of this manuscript. The authors, Dr. Muhammad Jamil and Arsalan Ahmed, are thoroughly obliged to the Department of Mathematics, NED University of Engineering \& Technology, Karachi-75270, Pakistan, and also would like to express their gratitude to the Higher Education Commission of Pakistan for assisting and facilitating this research work.

\section{Authors' contributions}

Dr. Muhammad Jamil gave the main idea and prepared the graphical part and their interpretations, and Arsalan Ahmed accomplished the mathematical calculations and wrote the manuscript. The manuscript was written through the contribution of all authors. The authors discussed the results, reviewed, and approved the final version of the manuscript.

\section{Funding}

Not applicable.

\section{Availability of data and materials}

Not applicable.

\section{Competing interests}

The authors declare that they have no competing interests.

Received: 13 July 2019 Accepted: 22 April 2020

Published online: 13 May 2020

\section{References}

1. Abdel-Rahman, G. M.: Effect of magnetohydrodynamic on thin films of unsteady micropolar fluid through a porous medium. J. Mod. Phys. 2, 1290-1304 (2011)

2. Ishak, A., Nazar, R., Pop, I.: Magnetohydrodynamic (MHD) flow and heat transfer due to a stretching cylinder. Energy Convers. Manag. 49, 3265-3269 (2008)

3. Shehzad, S. A., Hayat, T., Alsaedi, A.: Three-dimensional MHD flow of Casson fluid in porous medium with heat generation. J. Appl. Fluid Mech. 9, 215-223 (2016)

4. Khalid, A., Khan, I., Khan, A., Sha, S.: Influence of wall couple stress in MHD flow of a micropolar fluid in a porous medium with energy and concentration transfer. Results Phys. 9, 1172-1184 (2018)

5. Fatunmbi, E. O., Adeniyan, A.: MHD stagnation point-flow of micropolar fluids past a permeable stretching plate in porous media with thermal radiation chemical reaction and viscous dissipation. J. Adv. Math. Comput. Sci. 26, 1-19 (2018)

6. Hammouch, Z: Multiple solutions of steady MHD flow of dilatant fluids. arXiv preprint (2008). arXiv:0802.1851

7. Makinde, O. D., Khan, W. A., Khan, Z. H.: Analysis of MHD nanofluid flow over a convectively heated permeable vertical plate embedded in a porous medium. J. Nanofluids. 5(4), 574-580 (2016)

8. Makinde, O. D., Kumar, K. G., Manjunatha, S., Gireesha, B. J.: Effect of nonlinear thermal radiation on MHD boundary layer flow and melting heat transfer of micro-polar fluid over a stretching surface with fluid particles suspension. Defect Diffus. Forum. 378, 125-136 (2017)

9. Rundora, L., Makinde, O. D.: Analysis of unsteady MHD reactive flow of non-Newtonian fluid through a porous saturated medium with asymmetric boundary conditions. Iran. J. Sci. Technol. Trans. Mech. Eng. 40(3), 189-201 (2016)

10. Makinde, O. D., Reddy, M. G.: MHD peristaltic slip ow of Casson uid and heat transfer in channel filled with a porous medium. Sci. Iran. 26(4A), 2342-2355 (2019) 
11. Kumar, T. S., Kumar, B. R., Makinde, O. D., Kumar, A. G. V.: Magneto-convective heat transfer in micropolar nanofluid over a stretching sheet with non-unifrom heat source/sink. Defect Diffus. Forum. 387, 78-90 (2018)

12. Eringen, A. C.: Simple microfluids. Int. J. Eng. Sci. 2, 205-217 (1964)

13. Mekheimer, K. S., Abo-Elkhair, R. E., S.Husseny, Z.-A., Ali, A. T.: Similarity solution for flow of a micro-polar fluid through a porous medium. Appl. Appl. Math. 6, 341-352 (2011)

14. Raza, J., Rohni, A. M., Omar, Z:: A note on some solutions of micropolar fluid in a channel with permeable walls. Multidiscip. Model. Mater. Struct. 14, 91-101 (2017)

15. Hussanan, A., Zuki, M., llyas, S., Razman, K., Tahar, M.: Heat and mass transfer in a micropolar fluid with Newtonian heating: an exact analysis. Neural Comput. Appl. (2016). https://doi.org/10.1007/s00521-016-2516-0

16. Sheikholeslami, M., Hatami, M., Ganji, D. D.: Micropolar fluid flow and heat transfer in a permeable channel using analytical method. J. Mol. Liq. 194, 30-36 (2014)

17. Lukaszewicz, G.: Long time behavior of 2D micropolar fluid flows. Math. Comput. Model. 34, 487-509 (2001)

18. Ravi, S., Prasad, R. S.: Interaction of pulsatile flow on the peristaltic motion of couple stress fluid through porous medium in a flexible channel. Eur. J. PURE Appl. Math. 3, 213-226 (2010)

19. Pashaev, O., Tanoğlu, G.: Vector shock soliton and the hirota bilinear method. Chaos, Solitons Fractals. 26, 95-105 (2005)

20. Hirota, R.: Exact envelope-soliton solutions of a nonlinear wave equation. J. Math. Phys. 14, 805-809 (1973)

21. Dehghan, M., Manafian, J., Saadatmandi, A.: Solving nonlinear fractional partial differential equations using the homotopy analysis method. Numer. Methods Partial Differ. Equ. 26, 448-479 (2010)

22. Zhang, B. G., Li, S. Y., Liu, Z. R.: Homotopy perturbation method for modified Camassa-Holm and Degasperis-Procesi equations. Phys. Lett. A. 372, 1867-1872 (2008)

23. Shi, Y., Li, X., Zhang, B. G.: Traveling wave solutions of two nonlinear wave equations by $\left(G^{\prime} \backslash G\right)$ expansion method. Adv. Math. Phys. (2018). ID 8583418 8. https://doi.org/10.1155/2018/8583418

24. He, J., Abdou, M. A.: New periodic solutions for nonlinear evolution equations using Exp-function method. Chaos, Solitons Fractals. 34, 1421-1429 (2007)

25. He, J. H., Wu, X. H.: Exp-function method and its application to nonlinear equations. Chaos, Solitons Fractals. 38 903-910 (2008)

26. Islam, R., Khan, K.: Traveling wave solutions of some nonlinear evolution equations. Alex. Eng. J. 54, 263-269 (2015)

27. Iyiola, O. S., Folarin, S. B.: Approximate Analytical Study of Fingero-Imbibition phenomena of time-fractional type in double phase flow through porous media. Eur. J. PURE Appl. Math. 7, 210-229 (2014)

28. Khan, N. A., Ara, A., Jamil, M.: Traveling waves solution of a micropolar fluid. Int. J. Nonlinear Sc. Num. Simul. 10 $1121-1125$ (2009)

29. Khan, N. A., Mahmood, A., Jamil, M., Khan, N.-U.: Traveling wave solutions for MHD aligned flow of a second grade fluid. Int. J. Chm. React. Engg. 8, A163 (2010)

30. Khan, N. A., Ara, A., Jamil, M., Yildirim, A.: Traveling wave solutions for MHD aligned flow of a second grade fluid. A symmetry independent approach. J. King Saud Uni. Sc. 24, 63-67 (2011)

31. Khan, N. A., Khan, H.: Traveling wave solutions for $(3+1)$ dimensional equations arising in fluid mechanics. Nonlinear Eng. 3, 209-214 (2014)

32. Khan, N. A., Khan, H., Ali, S. A.: Exact solutions for MHD flow of couple stress fluid with heat transfer. J. Egyptain Math. Soc. 24, 125-129 (2016)

33. Zhao, Y., Chen, L., Zhang, X. R.: Traveling wave solutions to incompressible unsteady 2-D laminar flows with heat transfer boundary. Int. Commun. Heat Mass Transf. 75, 206-217 (2016)

34. Kucaba-Pietal, A.: Microchannels flow modelling with the micropolar fluid theory. Tech. Sci. 52, 209-2014 (2004)

\section{Publisher's Note}

Springer Nature remains neutral with regard to jurisdictional claims in published maps and institutional affiliations.

\section{Submit your manuscript to a SpringerOpen ${ }^{\circ}$ journal and benefit from:}

- Convenient online submission

- Rigorous peer review

- Open access: articles freely available online

- High visibility within the field

- Retaining the copyright to your article

Submit your next manuscript at $\gg$ springeropen.com 